Article

\title{
Irradiation Investigation: Exploring the Molecular Gas in NGC 7293
}

\author{
Jesse Bublitz ${ }^{1, * \mathbb{D}}$, Joel Kastner ${ }^{1} \mathbb{D}$, Pierre Hily-Blant ${ }^{2}$, Thierry Forveille ${ }^{2}$, \\ Miguel Santander-García ${ }^{3}$ (D), Valentin Bujarrabal ${ }^{3}$, Javier Alcolea ${ }^{3}$ and Rodolfo Montez, Jr. ${ }^{4}$ \\ 1 Center for Imaging Science, School of Physics \& Astronomy, and Laboratory for Multiwavelength \\ Astrophysics, Rochester Institute of Technology, Rochester, NY 14623, USA; jhkpci@cis.rit.edu \\ 2 Université Grenoble Alpes, CNRS, IPAG, 38000 Grenoble, France; \\ pierre.hily-blant@univ-grenoble-alpes.fr (P.H.-B.); thierry.forveille@univ-grenoble-alpes.fr (T.F.) \\ 3 Observatorio Astronómico Nacional, Instituto Geográfico Nacional, 28014 Madrid, Spain; \\ m.santander@oan.es (M.S.-G.); v.bujarrabal@oan.es (V.B.); j.alcolea@oan.es (J.A.) \\ 4 Smithsonian Astrophysical Observatory, Cambridge, MA 02138, USA; rodolfo.montez@cfa.harvard.edu \\ * Correspondence:jtb1435@rit.edu
}

Received: 1 March 2020; Accepted: 31 March 2020; Published: 8 April 2020

\begin{abstract}
Background: Many planetary nebulae retain significant quantities of molecular gas and dust despite their signature hostile radiation environments and energetic shocks. Photoionization and dissociation by extreme UV and (often) X-ray emission from their central stars drive the chemical processing of this material. Their well-defined geometries make planetary nebulae ideal testbeds for modeling the effects of radiation-driven heating and chemistry on molecular gas in photodissociation regions. Methods: We have carried out IRAM 30m/APEX 12m/ALMA radio studies of the Helix Nebula and its molecule-rich globules, exploiting the unique properties of the Helix to follow up our discovery of an anti-correlation between $\mathrm{HNC} / \mathrm{HCN}$ line intensity ratio and central star UV Luminosity. Results: Analysis of HNC/HCN across the Helix Nebula reveals the line ratio increases with distance from the central star, and thus decreasing incident UV flux, indicative of the utility of the $\mathrm{HNC} / \mathrm{HCN}$ ratio as a tracer of UV irradiation in photodissociation environments. However, modeling of the observed regions suggests $\mathrm{HNC} / \mathrm{HCN}$ should decrease with greater distance, contrary to the observed trend. Conclusion: $\mathrm{HNC} / \mathrm{HCN}$ acts as an effective tracer of $\mathrm{UV}$ irradiation of cold molecular gas. Further model studies are required.
\end{abstract}

Keywords: planetary nebulae; astrochemistry; molecules; NGC 7293; Helix Nebula

\section{Introduction}

The molecular chemistries of planetary nebulae $(\mathrm{PNe})$ provide ideal testbeds to explore the role that high-energy irradiation plays in photodissociation regions (PDRs). As the end-stage of $\sim 1-8 \mathrm{M}_{\odot}$ stars, the ejected gas and dust of a PN leaves behind the hot core of the dying central star (CSPN), which is a copious source of far-UV and X-ray photons [1]. The irradiation of the nebular envelope then drives the chemistry observed across PNe of varying morphologies. Analysis of the molecular features in this gas provides a means to probe the evolution of the $\mathrm{PN}$ as it distributes the material into the interstellar medium.

Two relatively abundant trace molecules, $\mathrm{HCN}$ and $\mathrm{HNC}$, vary by several orders of magnitude across a range of PN morphologies and ages (i.e., [2-4]). Early studies suggest the intertwined production and destruction of $\mathrm{HNC}$ and $\mathrm{HCN}$ are highly temperature-dependant [4,5], a hypothesis that has been further supported by recent observations of a correlation between $\mathrm{HNC} / \mathrm{HNC}$ and gas temperature in the Orion Bar [3]. Variation in the ratio has been observed across cold cloud core, 
protostellar, and protoplanetary environments in regions of $10^{1}-10^{2} \mathrm{~K}[6-8]$, demonstrating its potential utility as a probe of gas temperature in disparate astrophysical domains. Alternatively, however, the $\mathrm{HNC} / \mathrm{HCN}$ ratio may be governed by selective photodissociation of the molecules, wherein HNC is more readily dissociated by bright UV sources to form $\mathrm{HCN}$ than the inverse reaction [9].

The potential value of observations of molecule-rich PNe to our understanding of the processes governing the $\mathrm{HNC} / \mathrm{HCN}$ ratio has been overlooked in past works, though there is recent interest in the molecular ratio $[2,10]$. Driven by this recent progress, we seek a representative environment to establish whether and how UV emission from the CSPN and gas temperature affect the HNC/HCN ratio.

The expansive envelope ( $0.46 \mathrm{pc}$ radius) and intense CSPN emission of the highly evolved NGC 7293 (the Helix Nebula) provide such an environment. At a distance of 200 pc [11], the molecular envelope of the Helix covers $15-25^{\prime}$ arcminutes on the sky. Its CSPN is a strong UV source (89 $\left.L_{\odot},[12]\right)$, and displays point-like $X$-ray emission [1,13]. The Helix contains numerous dense, dusty, plasma-embedded neutral globules $\sim 0.15 \mathrm{pc}$ from its CSPN, as well as an extended envelope of clumpy molecular gas [14,15]. When taken as a whole, the Helix presents a factor $\sim 20$ gradient in UV flux from the inner globules to the edges of the molecular ring at $\sim 7.5^{\prime}$ (Bublitz et al., in prep), and hence is the best PN in which to establish how HNC/HCN varies across an individual PN.

In this proceedings paper, we present the current state of our radio molecular line observing campaign targeting the Helix Nebula and its globules, with the primary goal to gain insight into the mechanisms driving the $\mathrm{HNC} / \mathrm{HCN}$ ratio. We employ radiative transfer modeling to interpret these observations so as to constrain the molecular abundances and gas physical conditions within the Helix.

\section{Observations}

\subsection{Single-Dish Observations: IRAM $30 \mathrm{~m}$ and APEX $12 \mathrm{~m}$}

Six positions in the Helix Nebula have been observed with the Institut de Radioastronomie Millimétrique (IRAM) $30 \mathrm{~m}$ and Atacama Large Pathfinder Experiment (APEX) $12 \mathrm{~m}$ telescopes. At just $0.11-0.14$ pc away from the CSPN are three dense molecular knots (Globules A, B, and C), previously the focus of IRAM $30 \mathrm{~m}{ }^{12} \mathrm{CO}$ observations [15]. The Helix West position follows from a molecular line survey that observed multiple PNe [2], while the East position lies symmetrically opposite to the CSPN as a means to compare molecular and UV properties in the extended molecular envelope. The final "Helix Rim" position was derived from [10] so as to sample molecular gas at an intermediate distance. Observations were carried out in the Spring of 2018 and August 2019, respectively. All data were reduced and analyzed using the Continuum and Line Analysis Single-Dish Software ((CLASS; GILDAS version feb18c), publicly available through IRAM (Figure 1).

The Eight MIxer Receiver (EMIR) setup on the IRAM telescope allows for a dual-band configuration with pairs of $8 \mathrm{GHz}$ bandwidths per band across the $1 \mathrm{~mm}$ and $3 \mathrm{~mm}$ regimes. The observed frequency ranges cover 87-91, 109-117, 228-232, and 265-273 GHz, capturing a broad range of molecular emission lines.

Transitions of $\mathrm{HCN}, \mathrm{HCO}^{+}, \mathrm{HNC}, \mathrm{C}_{2} \mathrm{H}, \mathrm{CN},{ }^{13} \mathrm{CO}$, and ${ }^{12} \mathrm{CO}$ were identified across the six positions targeted in the Helix Nebula. The spectra of the Helix globules yielded the first detections of molecules other than ${ }^{12} \mathrm{CO}$ (Bublitz et al., in prep), demonstrating that they are molecule-rich in nature. Measurement of the $\mathrm{J}=1 \rightarrow 0$ and $\mathrm{J}=3 \rightarrow 2$ transitions of $\mathrm{HCN}$ and $\mathrm{HNC}$ in Globule B further assist in refining gas excitation properties. Line ratios of $\mathrm{HCN}$ hyperfine transitions indicate optically thin gas in the globules and Rim position, while the overlapping emission sources with nearby velocities in the East and West positions make the hyperfine structure difficult to interpret. 

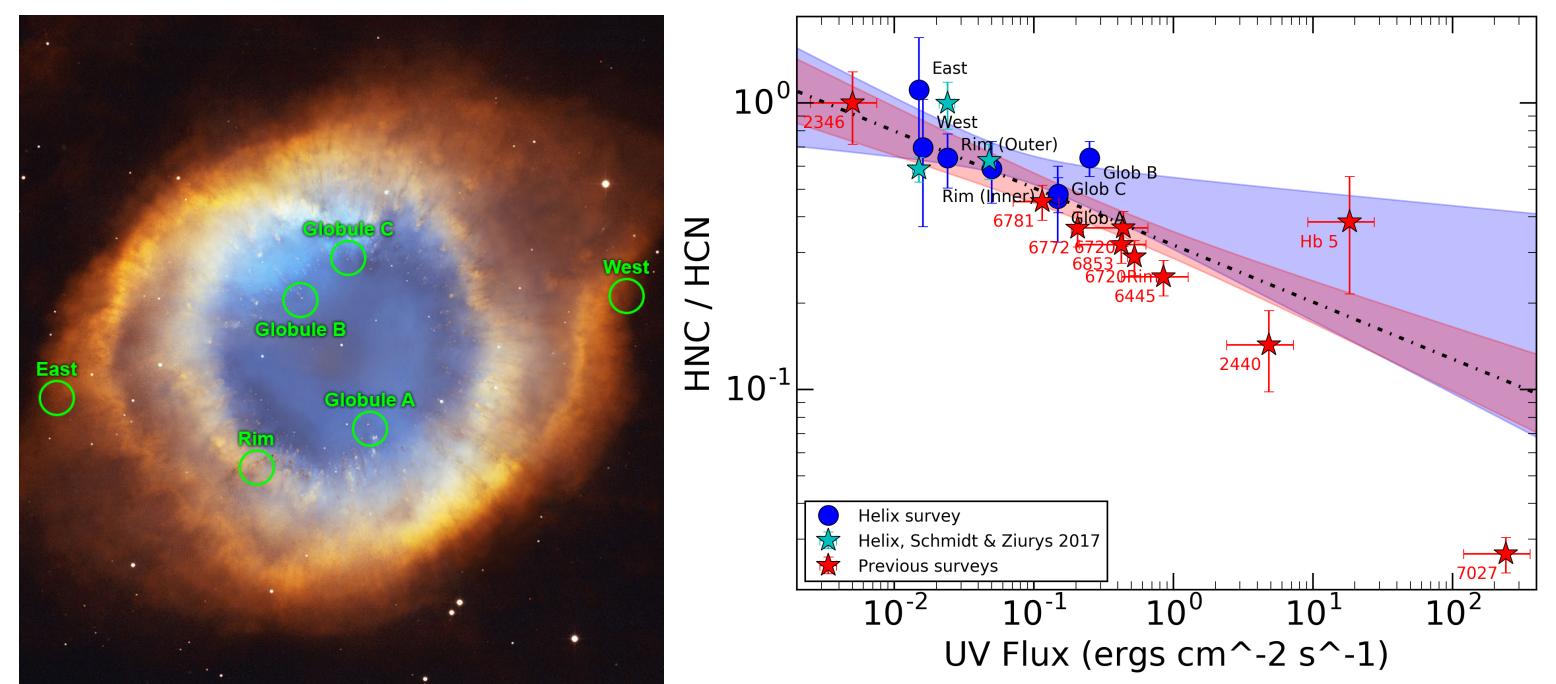

Figure 1. (Left) HST optical image of the Helix Nebula (NASA, NOAO, ESA, the Hubble Helix Nebula Team, M. Meixner (STScI), and T.A. Rector (NRAO)), with positions and beam widths of our $30 \mathrm{~m}$ observations indicated (circles). (Right) $\mathrm{HNC} / \mathrm{HCN}$ line intensity ratio vs. CSPN UV flux for the Helix Nebula and previous PNe survey sources. Linear regression fits to Helix survey data (blue) and entire data set (red) are displayed with $1 \sigma$ errors shaded.

Emission lines were fit with Gaussian functions to determine integrated line intensities. Computed $\mathrm{HNC} / \mathrm{HCN}$ line ratios range from 0.46-1.11. Using emission line radial velocities from the $30 \mathrm{~m}$ observations and modeling of the nebular expansion (e.g., [16,17]), we estimated the deprojected distance of each position from the CSPN. With the radii and CSPN $\mathrm{L}_{U V}$ ([2], and references therein), a calculation of the incident UV flux has been made on a given region of the nebula. In the right panel of Figure 1, we present the HNC/HCN ratio for the positions in the Helix, as well as other PNe in the literature where it is possible to compute the UV flux incident on the gas sampled by those observations.

In addition to the IRAM radio observations, the $\mathrm{J}=2 \rightarrow 1$ transitions of $\mathrm{HCN}$ and $\mathrm{HCO}^{+}$ (176-180 GHz) were observed across the same six Helix positions with the APEX $12 \mathrm{~m}$ telescope. These emission lines allowed us to better constrain gas models of the photodissociation regions in the nebula.

\subsection{ALMA}

Globules $B$ and $C$ were imaged in the $1 \mathrm{~mm}$ and $3 \mathrm{~mm}$ regimes with the Atacama Large Millimeter Array (ALMA) during Cycle 6. Data were reduced and preliminary analysis performed via CASA. Resulting images of ${ }^{12} \mathrm{CO}, \mathrm{HCN}, \mathrm{HCO}^{+}$, and $\mathrm{HNC}$ are presented in Figure 2. The globules appear highly filamentary in nature, with pronounced heads and $10-15^{\prime \prime}$ long tails trailing away from the central star. Distinct hotspots can be seen in $\mathrm{HCN} \mathrm{HCO}^{+}$, and $\mathrm{HNC}$, though the emission generally traces that of ${ }^{12} \mathrm{CO}$. The ${ }^{12} \mathrm{CO}$ image of Globule $\mathrm{C}$ was originally proposed by $\mathrm{P}$. Huggins in 2014, with analysis and publication performed contemporaneously with the new observations [18]. A detailed analysis of the imaged molecules and their analysis is forthcoming (Bublitz et al., in prep). 

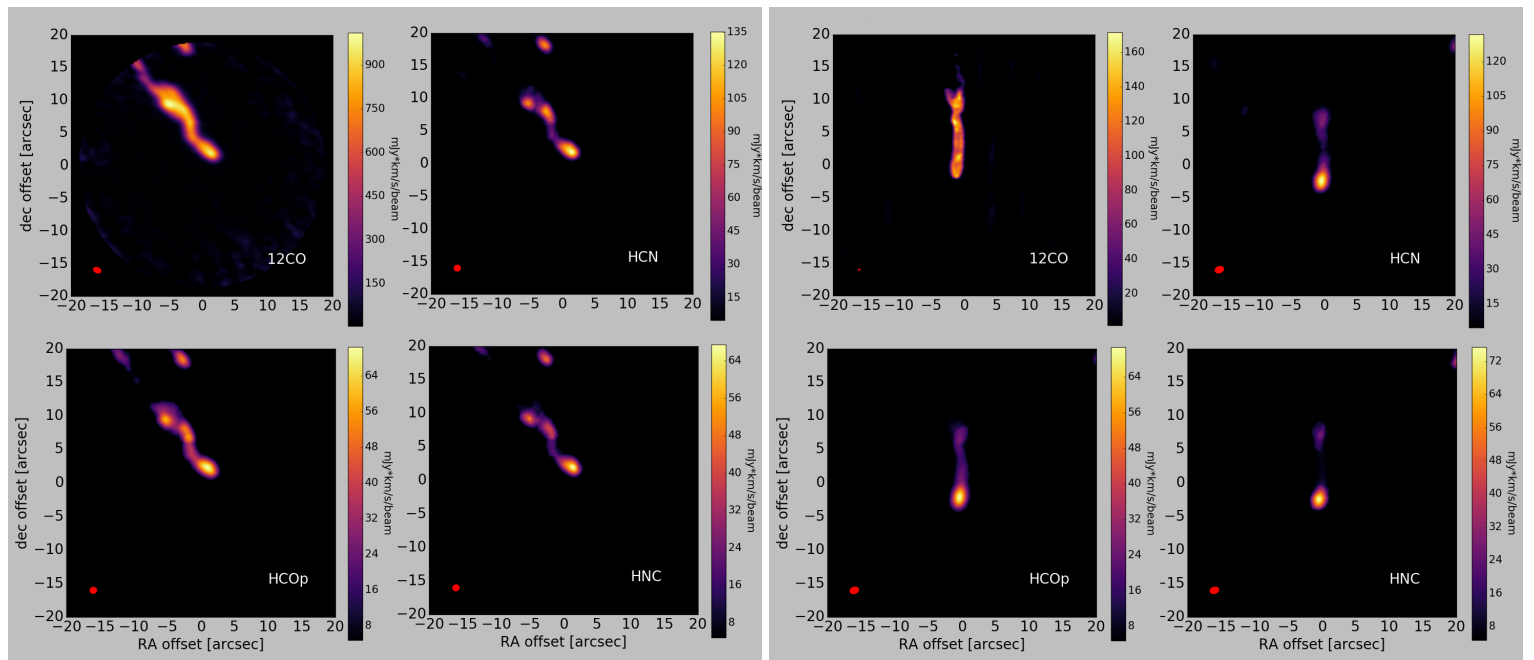

Figure 2. (Left) ALMA moment 0 image stamps of Globule $B$ in $1 \mathrm{~mm}$ and $3 \mathrm{~mm}$ transitions of ${ }^{12} \mathrm{CO}$, $\mathrm{HCN}, \mathrm{HCO}^{+}$, and $\mathrm{HNC}$. (Right) ALMA moment 0 image stamps of Globule $\mathrm{C}$ in the same molecular transitions as Globule B. CO image also published in [18].

\subsection{Modeling}

Modeling of Globule B was performed with RADEX, a publicly available, non-LTE radiative transfer code [19], where the line intensities of designated molecules are computed along a radiatively and collisionaly excited 1-D slab. All observed transitions of HCN and HNC are plotted as contours of the model output to identify solution spaces for a given molecule and temperature (Figure 3, left). The three transitions of $\mathrm{HCN}$ enabled a tight constraint on column density and the $\mathrm{H}_{2}$ density of the gas below $T_{k i n}=60 \mathrm{~K}$. HNC solutions remain degenerate for gas density, however the results indicate that $\mathrm{HNC}$ generally resides in a lower density region than $\mathrm{HCN}$.
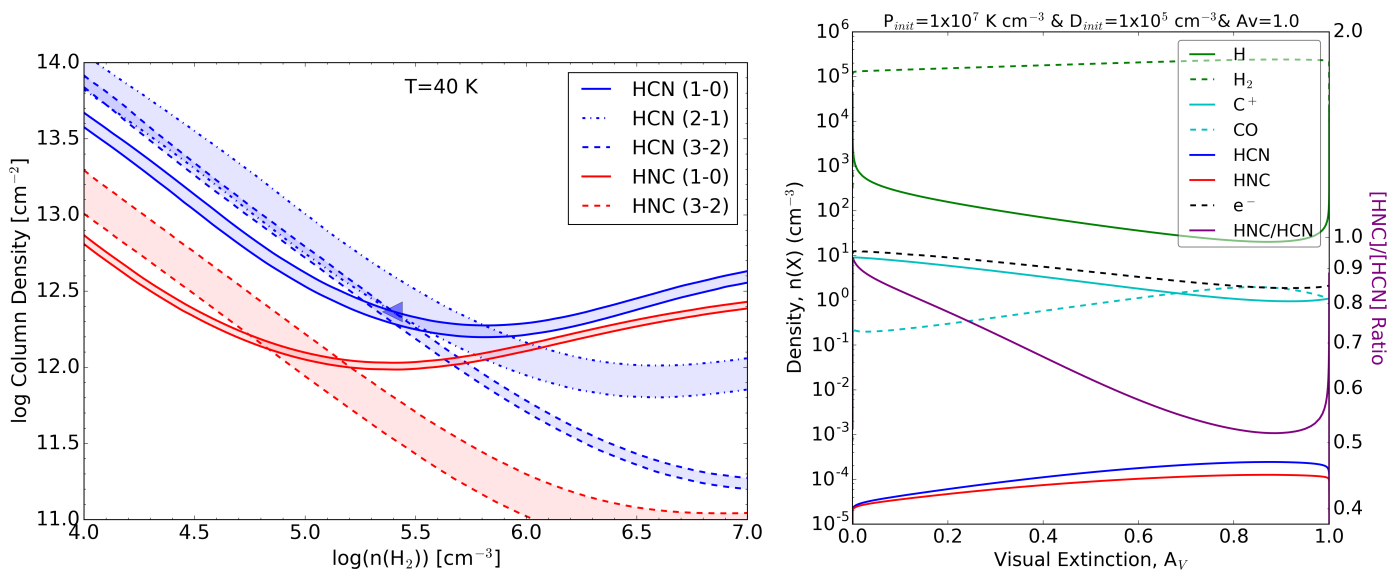

Figure 3. (Left) Sample RADEX predictions of molecular column and $\mathrm{H}_{2}$ number densities resulting in the observed ranges ( $1 \sigma$ uncertainties) of $\operatorname{HCN}$ and $\operatorname{HNC}(J=1 \rightarrow 0,2 \rightarrow 1,3 \rightarrow 2)$ line fluxes for Globule B, here assuming $T_{k i n}=40 \mathrm{~K}$. Regions of overlap reveal narrow solution spaces in $\mathrm{N}(\mathrm{HCN}$, $\mathrm{HNC}$ ) for temperatures below $60 \mathrm{~K}$, while $\mathrm{H}_{2}$ density remains degenerate for HNC. Convergence of all HCN transitions is marked (blue triangle). (Right) Meudon PDR modeling for a slab of gas with fixed pressure $\left(\mathrm{P}=10^{7} \mathrm{~K} \mathrm{~cm}^{-3}\right.$ ) confirms sub-unity $\mathrm{HNC} / \mathrm{HCN}$ (purple) at increased depth and thus decreased UV penetration within the model globule. A noted increase in HCN compared to HNC (or decreasing $\mathrm{HNC} / \mathrm{HCN}$ ) at greatest depth of the slab contradicts the trend in Figure 1, suggesting an incomplete understanding of the chemical processes within the Helix globules. We have also plotted densities for additional species such as $\mathrm{H}_{\text {and }} \mathrm{C}^{+}$(green and light blue, respectively) that are commonly used as tracers of irradiation, density, etc. 
To better understand the structure of the PDR environment in the globules and molecular envelope, the radiative transfer Meudon code was implemented for a globule-like slab of gas irradiated by a model WD spectrum, scaled to the flux of the Helix CSPN [20]. The code then calculates the heating, cooling, and reaction rates for a catalog of atomic and molecular species. Abundances for relevant species are plotted across the $1 \mathrm{~A}_{V}$-scale region (Figure 3, right).

\section{Discussion}

Comparison of the $\mathrm{HNC} / \mathrm{HCN}$ line ratio with the computed UV flux of the Helix Nebula reveals an anticorrelation that is consistent with the results of [2]. The addition of PNe with established UV flux from published literature sources further extends the trend of decreasing $\mathrm{HNC} / \mathrm{HCN}$ into the regime where UV flux is strongest, suggesting that the ratio acts as a powerful tracer of UV irradiation in PDR environments.

RADEX modeling of Globule B yields column density estimates for both molecules, but hints at an effect that is contrary to the observed results. That is, HCN appears to reside in regions of higher number density, hence perhaps closer to the cores of the globules, rather than along the globule surfaces where it is expected to be preferentially produced by UV emission. Similarly, the Meudon models predict that while both HCN and HNC rise in density towards the core of the globule where UV irradiation is weakest, the relative abundance HNC/HCN decreases along this path. Thus both modeling approaches suggest that within an individual region of gas, the expected anticorrelation between HNC/HCN and UV flux is inverted, with HCN preferentially residing deeper in the core than its isomer HNC. In forthcoming analysis (Bublitz et al., in prep), we will explore potential solutions to this conundrum, by more fully investigating the possible model parameter spaces that might describe molecular gas in the Helix nebula.

Author Contributions: Conceptualization, J.B. and J.K.; methodology, J.B.; software, J.B.; validation, n.a.; formal analysis, J.B.; investigation, J.B.; resources, n.a.; data curation, J.B.; writing-original draft preparation, J.B.; writing-review and editing, J.K., P.H.-B., T.F., M.S.-G., V.B., J.A., and R.M.; visualization, J.B.; supervision, J.K.; project administration, J.K.; funding acquisition, J.K., M.S.-G., V.B., and J.A. All authors have read and agreed to the published version of the manuscript.

Funding: This research is supported by Space Telescope Science Institute grant HST-GO-15953.001-A to RIT. Santander-García, Bujarrabal, and Alcolea acknowledge support from the Spanish Science and Innovation Ministry (MICINN) research grant AYA2016-78994-P.

Acknowledgments: This work is based on observations carried out under project number 109-17 with the IRAM $30 \mathrm{~m}$ telescope, data acquired with the Atacama Pathfinder Experiment (APEX) under program ID 0103.D-0682, and makes use of the following ALMA data: ADS/JAO.ALMA\#2018.1.01006.S, ADS/JAO.ALMA\#2012.1.00116. IRAM is supported by INSU/CNRS (France), MPG (Germany) and IGN (Spain). ALMA is a partnership of ESO (representing its member states), NSF (USA) and NINS (Japan), together with NRC (Canada), MOST and ASIAA (Taiwan), and KASI (Republic of Korea), in cooperation with the Republic of Chile. The Joint ALMA Observatory is operated by ESO, AUI/NRAO and NAOJ.

Conflicts of Interest: The authors declare no conflict of interest.

\section{References}

1. Kastner, J.H.; Montez, R., Jr.; Balick, B.; Frew, D.J.; Miszalski, B.; Sahai, R.; Blackman, E.; Chu, Y.-H.; Marco, O.D.; Frank, A.; et al. The CHANDRA X-ray survey of planetary nebulae (ChanPlaNS): Probing binarity, magnetic fields, and wind collisions. Astron. J. 2012, 144, 58.

2. Bublitz, J.; Kastner, J.H.; Santander-García, M.; Bujarrabal, V.; Alcolea, J.; Montez, R. A new radio molecular line survey of planetary nebulae- $\mathrm{HNC} / \mathrm{HCN}$ as a diagnostic of ultraviolet irradiation. Astron. Astrophys. 2019, 625, A101. [CrossRef]

3. Hacar, A.; Bosman, A.D.; van Dishoeck, E.F. HCN-to-HNC intensity ratio: A new chemical thermometer for the molecular ISM. Astron. Astrophys. 2020, 635, A4. [CrossRef]

4. Schilke, P.; Walmsley, C.M.; Pineau Des Forets, G.; Roueff, E.; Flower, D.R.; Guilloteau, S. A study of HCN, HNC and their isotopomers in OMC-1. I-Abundances and chemistry. Astron. Astrophys.1992, 256, 595. 
5. Graninger, D.M.; Herbst, E.; Öberg, K.I.; Vasyunin, A.I. The HNC/HCN ratio in star-forming regions. Astrophys. J. 2014, 787, 74. [CrossRef]

6. Graninger, D.; Öberg, K.I.; Qi, C.; Kastner, J. HNC in protoplanetary disks. Astron. Astrophys. Lett. 2015, 807, L15. [CrossRef]

7. Hirota, T.; Yamamoto, S.; Mikami, H.; Ohishi, M. Abundances of HCN and HNC in dark cloud cores. Astrophys. J. 1998, 503, 717. [CrossRef]

8. Jin, M.; Lee, J.-E.; Kim, K.-T. The HCN/HNC abundance ratio toward different evolutionary phases of massive star formation. Astrophys. J. 2015, 219, 1. [CrossRef]

9. Aguado, A.; Roncero, O.; Zanchet, A.; Agúndez, M.; Cernicharo, J. The Photodissociation of HCN and HNC: Effects on the HNC/HCN Abundance Ratio in the Interstellar Medium. Astrophys. J. 2017, 838, 33. [CrossRef] [PubMed]

10. Schmidt, D.R.; Ziurys, L. New detections of HNC in planetary nebulae: Evolution of the [HCN]/[HNC] ratio. Astrophys. J. 2017, 835, 79. [CrossRef]

11. Gaia Collaboration; Brown, A. G. A.; Vallenari, A.; Prusti, T.; de Bruijne, J. H. J.; Babusiaux, C.; Bailer-Jones, C. A. L.; Biermann, M. Evans, D. W.; Eyer, L.; Jansen, F. et al. Gaia Data Release 2 Summary of the contents and survey properties. Astron. Astrophys. 2018, 616, A1

12. Montez, R., Jr.; Kastner, J.H.; Balick, B.; Behar, E.; Blackman, E.; Bujarrabal, V.; Chu, Y.-H.; Corradi, R.L.M.; Marco, O.D.; Frank, A.; et al. The CHANDRA planetary nebula survey (ChanPlaNS). III. X-ray emission from the central stars of planetary nebulae. Astrophys. J. 2015, 800, 8. [CrossRef]

13. Guerrero, M.A.; Chu, Y.-H.; Gruendl, R.A.; Williams, R.M.; Kaler, J.B. The enigmatic x-ray point sources at the central stars of ngc 6543 and ngc 7293. Astrophys. J. 2001, 553, L55. [CrossRef]

14. Hora, J.L.; Latter, W.B.; Smith, H.A.; Marengo, M. Infrared observations of the helix planetary nebula. Astrophys. J. 2006, 652, 426. [CrossRef]

15. Huggins, P.J.; Bachiller, R.; Cox, P.; Forveille, T. CO in the cometary globules of the Helix nebula. Astrophys. J. 1992, 401, L43. [CrossRef]

16. Meaburn, J.; Clayton, C.A.; Bryce, M.; Walsh, J. R.; Holloway, A. J.; Steffen, W. The nature of the cometary knots in the helix planetary nebula (NGC 7293). MNRAS, 1998, 294, 201 [CrossRef]

17. Young, K.; Cox, P.; Huggins, P.; Forveille, T.; Bachiller, R. The molecular envelope of the helix nebula. Astrophys. J. 1999, 522, 387 [CrossRef]

18. Andriantsaralaza, M.; Zijlstra, A.A.; Avison, A. CO in the C1 globule of the Helix nebula with ALMA. MNRAS 2019, 491, 758 [CrossRef]

19. van der Tak, F.F.S.; Black, J.H.; Schöier, F.L.; Jansen, D.J.; van Dishoeck, E.F. A computer program for fast non-LTE analysis of interstellar line spectra with diagnostic plots to interpret observed line intensity ratios. Astron. Astrophys. 2007, 468, 627 [CrossRef]

20. Levenhagen, R.S.; Diaz, M.P.; Coelho, P.R.T.; Hubney, I. A grid of synthetic spectra for hot da white dwarfs and its application in stellar population synthesis. Astrophys. J. Suppl. Ser. 2017, 231, 1 [CrossRef]

(C) 2020 by the authors. Licensee MDPI, Basel, Switzerland. This article is an open access article distributed under the terms and conditions of the Creative Commons Attribution (CC BY) license (http://creativecommons.org/licenses/by/4.0/). 